\title{
Comparison of mortality according to hemorrhage control methods performed for hemodynamically unstable patients with traumatic pelvic fractures: $A$ multi-center study
}

\section{Myoung Jun Kim}

Wonju Severance Christian Hospital https://orcid.org/0000-0002-6421-2112

\section{Seung Hwan Lee}

Gachon University Gil Medical Center

Ji Young Jang

National Health Insurance Corporation Ilsan Hospital

Jae Gil Lee ( $\sim$ JAKII@yuhs.ac)

Yonsei University College of Medicine https://orcid.org/0000-0002-1148-8035

\section{Research article}

Keywords: Pelvic fracture, Hemorrhage control methods, Hemodynamic instability

Posted Date: May 27th, 2020

DOI: https://doi.org/10.21203/rs.3.rs-30761/v1

License: (c) (1) This work is licensed under a Creative Commons Attribution 4.0 International License.

Read Full License 
2 Comparison of mortality according to hemorrhage

3 control methods performed for hemodynamically

4 unstable patients with traumatic pelvic fractures: A

\section{5 multi-center study}

${ }^{1}$ Department of Surgery, Yonsei University Wonju College of Medicine, Wonju Severance Christian Hospital, Wonju, Republic of Korea

${ }^{2}$ Department of Traumatology, Gachon University Gil Medical Center, Incheon, Republic of Korea

${ }^{3}$ Department of Surgery, National Health Insurance Service Ilsan Hospital, Goyang, Republic of Korea

*Corresponding author:

Jae Gil Lee

Department of Surgery, Yonsei University College of Medicine 
Background: We analyzed the effects of hemorrhage control methods on the mortality of patients with hemodynamic instability due to pelvic fracture and investigated independent risk factors for mortality in these patients.

Methods: A total of 97 pelvic bone fracture patients with hemodynamic instability who visited the emergency departments of two university hospitals from January 2013 to October 2017 were enrolled. These patients were divided into the survival and non-survival groups based on 28-day mortality and acute hemorrhage mortality. Clinical data including laboratory test results, trauma severity scores, and hemorrhage control modalities were collected and statistically analyzed. Furthermore, the effects of each hemorrhagic control modality on mortality were analyzed. Multivariate logistic regression was performed to determine independent risk factors for mortality.

Results: Overall mortality was $41.24 \%$ (40 patients), 28-day mortality was $38.14 \%$ (37 patients), and acute hemorrhage mortality was $28.86 \%$ (28 patients). With respect to hemorrhage control modalities, 47 patients (48.5\%) underwent pelvic angiography, 45 patients $(46.4 \%)$ underwent pre-peritoneal pelvic packing, and 19 patients (19.6\%) underwent external fixation. Differences in hemorrhage control methods had no significant effects on mortality. However, there was a significant difference in mortality between the groups with and without any hemorrhage control methods. A multivariate logistic regression analysis revealed that patient age, trauma and injury severity score (TRISS; probability of survival), and transfusion amount within 24 hours were independent factors for 28-day mortality. Using the same statistical analysis, patient age, transfusion amount within 24 hours, Glasgow coma scale (GCS) score, and systolic blood pressure (SBP) were independent factors for mortality due to acute hemorrhage.

Conclusions: Hemorrhage control methods can aid in reducing acute hemorrhage mortality in hemodynamically unstable patients with pelvic fractures; nevertheless, no significant difference in mortality according to different hemorrhage control methods was found. Older age, higher 
transfusion requirements, low TRISS scores, GCS scores, and SBP are independent risk factors for mortality in pelvic fracture patients with hemodynamic instability.

\section{Background}

51 The treatment of traumatic pelvic fractures is a major challenge for surgeons in trauma units worldwide. Several studies have reported mortality rates of $50-60 \%$ for patients with traumatic pelvic fractures and hemodynamic instability [1, 2]. Hemodynamic instability results from massive bleeding from arteries, veins, and/or the fractured bone itself, and blood loss may quickly become life-threatening [3,4]. Therefore, appropriate and rapid treatment is required. It is critical to choose the best therapeutic approach for patients based on risk factors that may predispose them to critical situations.

There are several established methods for hemorrhage control with pelvic fractures, such as angiography and angio-embolization (AE), pre-peritoneal pelvic packing (PPP), and external fixation (EF). These methods are routinely performed at many institutions and have been successful for the resuscitation of patients with pelvic fractures and hemodynamic instability [5,6]. However, despite these efforts, pelvic fracture remains a life-threatening condition when the patient is hemodynamically unstable. Furthermore, patient outcomes differ between institutions and regions.

This study investigated the effects of different hemorrhage control methods on the survival of pelvic fracture patients with hemodynamic instability. Furthermore, we investigated independent risk factors potentially associated with mortality in this patient population. 
We conducted a retrospective observational study at two tertiary institutions in Seoul and Wonju in South Korea from January 2013 to October 2017. We reviewed the medical records of patients who visited the emergency department with traumatic pelvic fractures. Of these, patients with hemodynamic instability were included. Patients without pelvic fractures, patients younger than age 18 years, and those with pelvic fractures who were hemodynamically stable were excluded from the study. As a result, a total of 97 patients with traumatic pelvic fractures and hemodynamic instability were enrolled. Hemodynamic instability in adults was defined as systolic blood pressure (SBP) $<90 \mathrm{mmHg}$ on admission with evidence of skin vasoconstriction (cool and clammy skin, decreased capillary refill), altered level of consciousness, and/or shortness of breath or as SBP $>90 \mathrm{mmHg}$ but requiring bolus infusions/transfusions and/or vasopressor drugs and/or admission base excess $(\mathrm{BE})>-5 \mathrm{mmol} / \mathrm{L}$ and/or shock index $>1$ and/or transfusion requirement of at least 4-6 units of packed red blood cells within the first 24 hours [7].

Initially, we divided all patients into those who survived up to 28 days (28-day survival group) and those who did not (non-survival group). We then compared the clinical variables between these two groups. Additionally, we divided patients into those who died of acute hemorrhage due to their pelvic bone fracture and those who did not. Next, we compared the clinical variables between those two groups. The percentages of the hemorrhage control methods performed at two institutions were compared. Patient outcomes, such as mortality, were also compared.

This study was conducted in accordance with the Declaration of Helsinki and was approved by the Institutional Review Boards of Severance Hospital, Yonsei University Health System (4-2019-0761) and Wonju Severance Christian Hospital (CR319120), which waived the requirement for the acquisition of informed consent from patients because of the retrospective nature of the study.

\section{Clinical variables}

We analyzed sex, age, injury mechanism, vital signs, Glasgow coma scale (GCS) score, current medication, transfusion amount within the first 24 hours, abbreviated injury scale (AIS) score, injury 
severity score (ISS), revised trauma score (RTS), trauma and injury severity score (TRISS), Acute Physiology and Chronic Health Evaluation (APACHE) II score, arterial blood pH, BE levels, and lactate levels. Furthermore, pelvic radiography and abdomen-pelvic computed tomography was performed for almost all patients; patterns of pelvic fracture were classified as lateral compression 1 to 3 , anteriorposterior compression 1 to 3 , and vertical shearing according to the Young-Burgess classification $[8,9]$. The classification was based on the findings of trauma surgeons and orthopedic surgeons; it was additionally confirmed by surgery and radiology records. The types of hemorrhagic control modalities (pelvic binder wearing, angiography, AE, PPP, and EF) were investigated. We also investigated whether intraperitoneal surgery was performed in conjunction with hemorrhagic control of the pelvic bone fracture.

\section{Statistical analysis}

Statistical analysis of the investigated items was performed using SPSS Statistics 23.0 (IBM Corp., Armonk, NY). Categorical data are presented as numbers (\%) and were compared using the chi-square test or Fisher's exact test. Continuous variables are expressed as mean \pm standard deviation or as median and interquartile range, and data were compared between groups using Student's $t$-test or the Mann-Whitney $U$ test. Univariate analysis revealed the factors significantly associated with the need for hemorrhage control intervention, and these were included in the multivariate analysis. Logistic regression modeling was performed using the maximum likelihood method and backward stepwise selection. Goodness-of-fit was assessed using the Hosmer-Lemeshow test. Odds ratios (ORs) are provided with 95\% confidence intervals (CIs); $p<0.05$ was considered statistically significant.

\section{Results}

\section{Comparisons of baseline characteristics of patients}

The baseline characteristics of patients in both groups are summarized in Table 1. 
Table 1 Baseline characteristics of patients

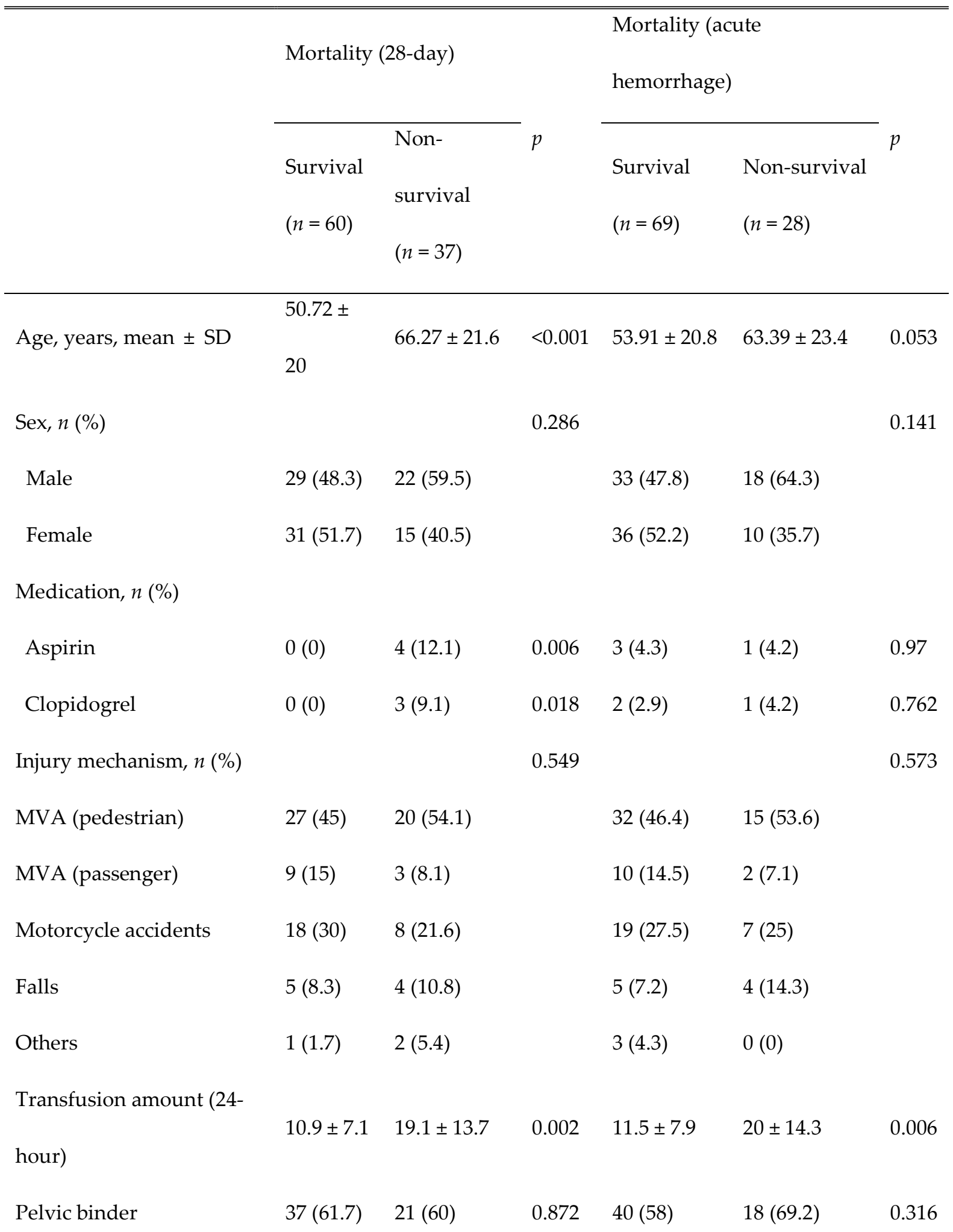


$\begin{array}{lllllll}\text { Combined laparotomy } & 10(16.7) & 10(27) & 0.221 & 11(15.9) & 9(32.1) & 0.074\end{array}$

SD, standard deviation; MVA, motor vehicle accident

Of the 97 patients, 37 (38.1\%) died within 28 days. The mean age at the time of accident among the surviving patients was $50.72 \pm 20$ years, which was statistically significantly different from the mean age of $66.27 \pm 21.6$ years for patients who died. Furthermore, statistically significant differences in the number of patients receiving aspirin $(p=0.006)$ or clopidogrel $(p=0.018)$ and the number of blood transfusions performed within the first 24 hours $(p=0.002)$ were observed between the two groups.

When categorized according to the injury mechanism, motor vehicle accidents involving pedestrians were the most frequent, followed by motorcycle accidents. Nevertheless, no statistically significant difference in the injury mechanism was noted between the two groups $(p=0.549)$. There was no significant difference between the 28-day survival group and non-survival group with respect to whether a pelvic binder was worn at the time of the patient's visit. Whether the patient underwent intraperitoneal surgery during treatment did not significantly affect 28-day mortality.

In the second analysis, 29 out of the 97 patients (29.9\%) died of acute hemorrhage associated with pelvic fracture (Table 1). The analysis showed no statistically significant between-group difference in age $(p=0.053)$ or medications such as aspirin $(p=0.97)$ and clopidogrel $(p=0.762)$. With respect to the injury mechanism, mortality was most frequently caused by acute hemorrhage due to motor vehicle accidents involving pedestrians, followed by injuries resulting from motorcycle accidents. Nonetheless, no significant between-group difference in the injury mechanism was found ( $p=0.573)$. The transfusion requirement within 24 hours was significantly different between the two groups $(p=0.006)$. was worn at the time of the patient's visit. Whether the patient underwent intraperitoneal surgery during treatment did not significantly affect acute hemorrhage mortality; however, it did show a trend toward significance. 
142 The trauma-related scores of patients are presented in Table 2.

Table 2 Comparison of trauma-related scores

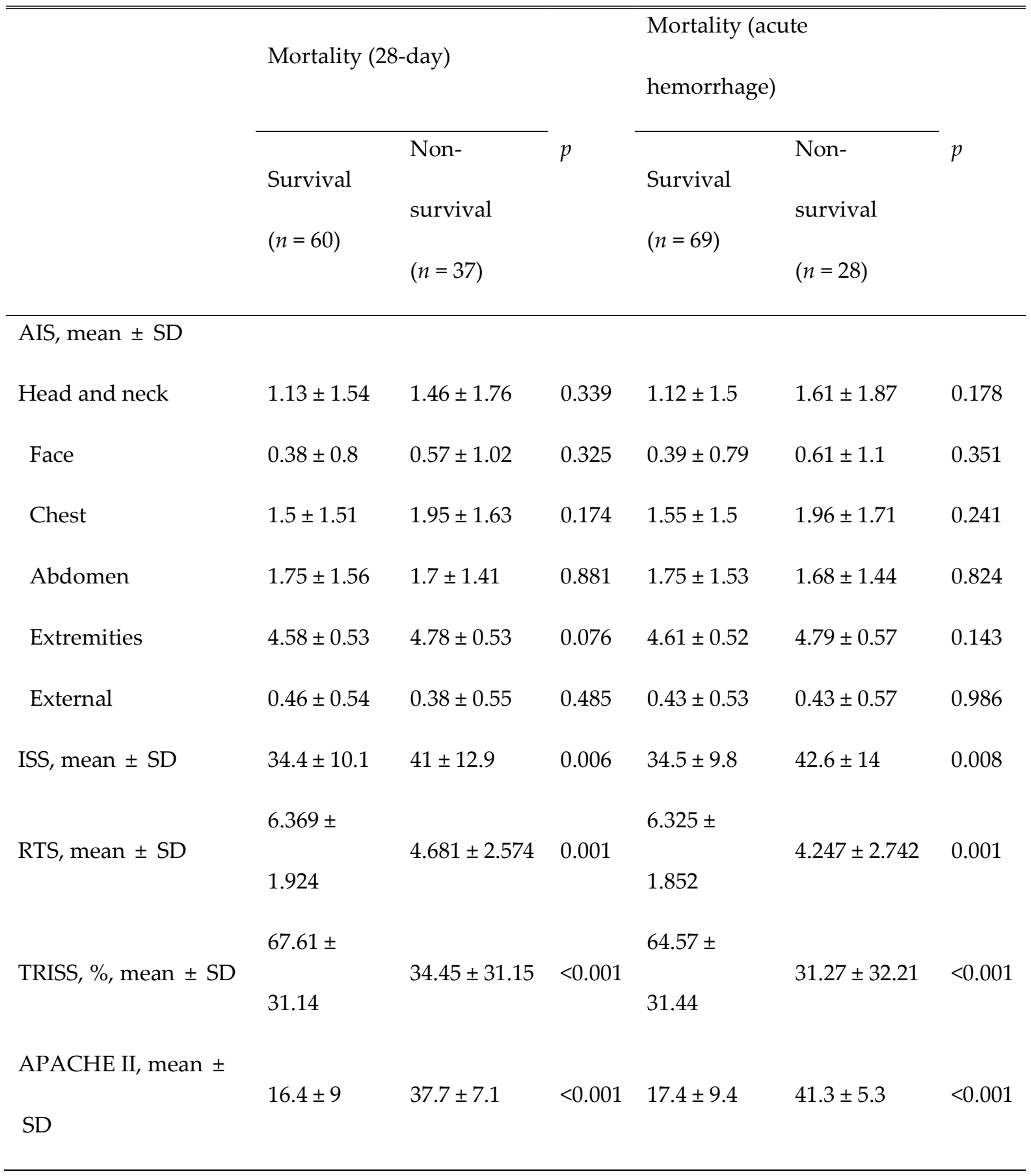


AIS, abbreviated injury scale; SD, standard deviation; ISS, injury severity score; RTS, revised trauma score; TRISS, trauma and injury severity score; APACHE, Acute Physiology and Chronic Health Evaluation mortality group. In contrast, the ISS $(p=0.006 ; p=0.008)$, RTS $(p=0.001 ; p=0.001)$, and TRISS $(p<0.001, p$

$150<0.001)$ were significantly different for both mortality criteria. The APACHE II score $(p<0.001 ; p<0.001)$

151 was also significantly different between these groups.

\section{Comparison of clinical variables between groups}

153 The vital signs and laboratory variables of patients are shown in Table 3.

Table 3 Comparison of clinical parameters between groups

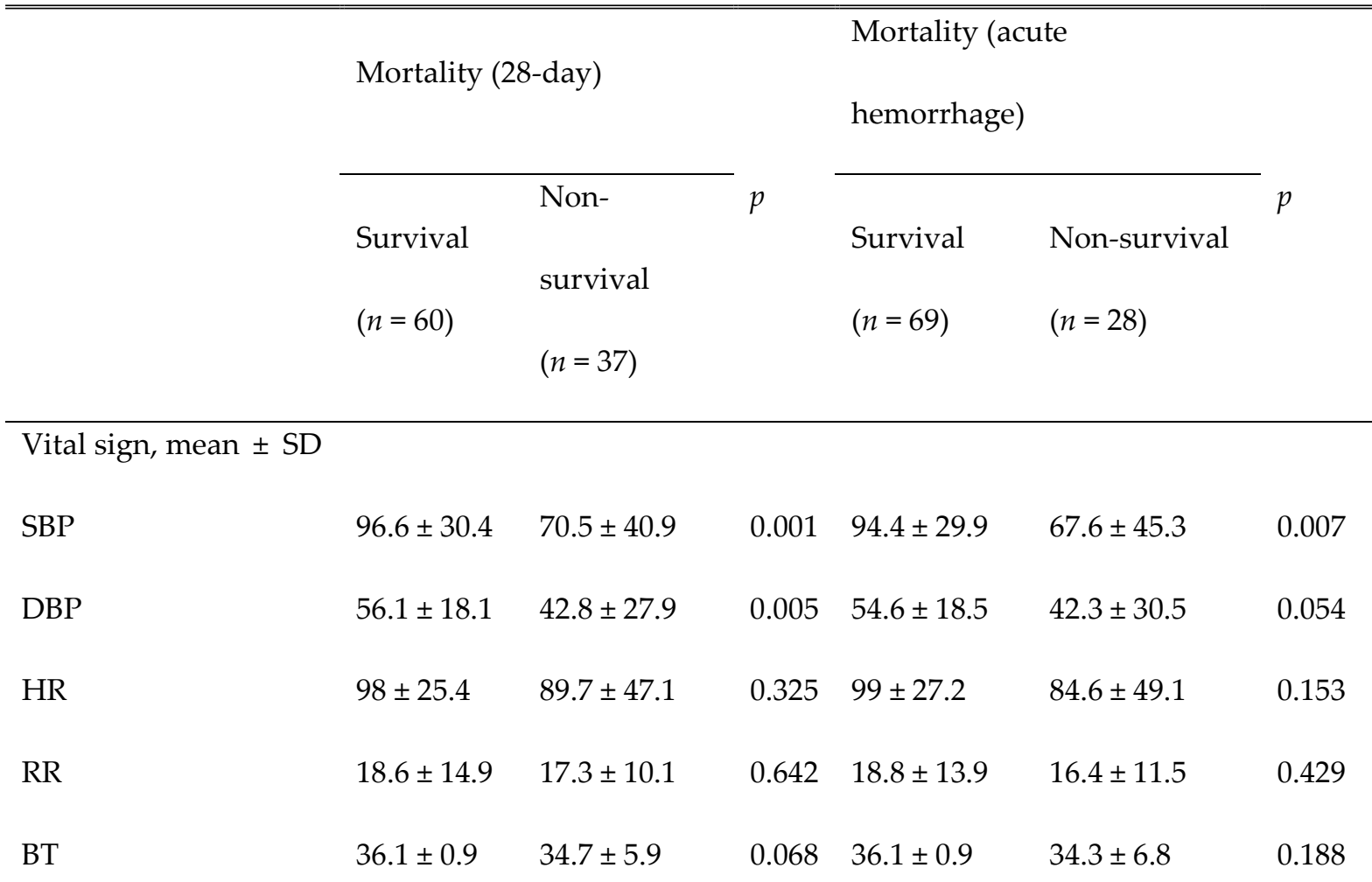




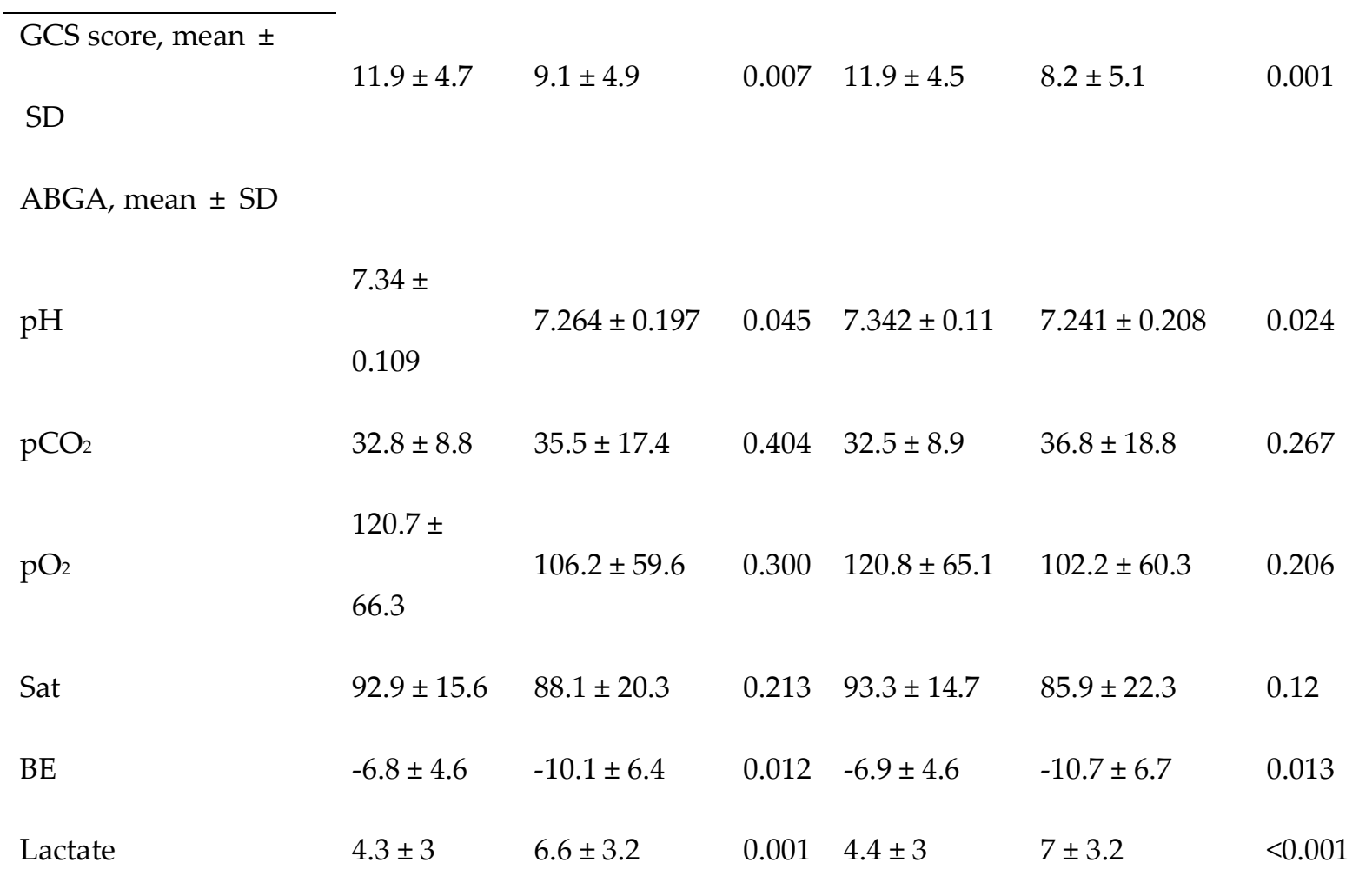

156 SBP, systolic blood pressure; DBP, diastolic blood pressure; HR, heart rate; RR, respiration rate; BT, body

157 temperature; GCS, Glasgow coma scale; ABGA, arterial blood gas analysis; BE, base excess

With respect to clinical variables, patients who died within 28 days or from acute hemorrhage were found to have significantly lower SBP $(p=0.001 ; p=0.007)$, diastolic blood pressure $(p=0.005 ; p=0.054)$, and GCS ( $p=0.007 ; p=0.001)$ than the surviving patients. Arterial blood gas analysis showed that patients who died within 28 days or from acute hemorrhage had statistically significantly higher BE $(p=0.012 ; p=$ $0.013)$ and higher lactate levels $(p=0.001 ; p<0.001)$ than surviving patients.

\section{Comparison of hemorrhage control methods between groups}

Before comparing mortality between groups, we investigated the percentage difference between the two institutions regarding each hemorrhage control method. We also investigated whether there were significant differences in mortality between the two institutions. As a result, the percentage of each 
169

170

171

172

173

174

175

\begin{tabular}{|c|c|c|c|c|c|c|}
\hline & \multicolumn{3}{|c|}{ Mortality (28-day) } & \multicolumn{2}{|c|}{$\begin{array}{l}\text { Mortality (acute } \\
\text { hemorrhage) }\end{array}$} & \multirow[b]{2}{*}{$p$} \\
\hline & $\begin{array}{l}\text { Survival } \\
(n=60)\end{array}$ & $\begin{array}{l}\text { Non- } \\
\text { survival } \\
(n=37)\end{array}$ & $p$ & $\begin{array}{l}\text { Survival } \\
(n=69)\end{array}$ & $\begin{array}{l}\text { Non-survival } \\
(n=28)\end{array}$ & \\
\hline Angiography & $33(55.9)$ & $14(38.9)$ & 0.107 & $37(54.4)$ & $10(37.0)$ & 0.127 \\
\hline $\begin{array}{l}\text { Angiography + } \\
\text { embolization }\end{array}$ & $23(38.3)$ & $9(24.3)$ & 0.154 & $25(36.2)$ & $7(25)$ & 0.286 \\
\hline PPP & $26(43.3)$ & $19(51.4)$ & 0.442 & $33(47.8)$ & $12(42.9)$ & 0.657 \\
\hline EF & $14(23.3)$ & $5(13.5)$ & 0.237 & $18(26.1)$ & $1(5.3)$ & 0.011 \\
\hline Angiography or PPP or EF & $49(81.7)$ & $27(73)$ & 0.313 & $58(84.1)$ & $18(64.3)$ & 0.032 \\
\hline
\end{tabular}

mortality $(p=0.081)$ and acute hemorrhage mortality $(p=0.371)$ rates did not show a significant difference between the two institutions.

We analyzed the differences in mortality for the different hemorrhage control methods of angiography, AE, PPP, and EF between groups (Table 4-1). We also compared the effects of these hemorrhage control measures on patient mortality (Table 4-2).

Table 4-1 Effects of hemorrhage control methods on patient mortality 


\begin{tabular}{|c|c|c|c|c|c|c|}
\hline & \multicolumn{2}{|c|}{ Mortality (28-day) } & \multirow[b]{2}{*}{$p$} & \multicolumn{2}{|c|}{$\begin{array}{l}\text { Mortality (acute } \\
\text { hemorrhage) }\end{array}$} & \multirow[b]{2}{*}{$p$} \\
\hline & $\begin{array}{l}\text { Survival } \\
(n=49)\end{array}$ & $\begin{array}{l}\text { Non- } \\
\text { survival } \\
(n=27)\end{array}$ & & $\begin{array}{l}\text { Survival } \\
(n=58)\end{array}$ & $\begin{array}{l}\text { Non-survival } \\
(n=18)\end{array}$ & \\
\hline $\begin{array}{l}\text { Hemorrhage control } \\
\text { methods }\end{array}$ & & & 0.259 & & & 0.350 \\
\hline Angiography alone & $19(38.8)$ & $6(22.2)$ & & $19(32.8)$ & $6(33.3)$ & \\
\hline PPP alone & $9(18.4)$ & $12(44.4)$ & & $13(22.4)$ & $8(44.4)$ & \\
\hline EF alone & $1(2)$ & $0(0)$ & & $1(1.7)$ & $0(0)$ & \\
\hline Angiography + PPP & $7(14.3)$ & $4(14.8)$ & & $8(13.8)$ & $3(16.7)$ & \\
\hline Angiography + EF & $3(6.1)$ & $2(7.4)$ & & $5(8.6)$ & $0(0)$ & \\
\hline $\mathrm{PPP}+\mathrm{EF}$ & $6(12.2)$ & $1(3.7)$ & & $7(12.1)$ & $0(0)$ & \\
\hline Angiography + PPP + EF & $4(8.2)$ & $2(7.4)$ & & $5(8.6)$ & $1(5.6)$ & \\
\hline
\end{tabular}

PPP, preperitoneal pelvic packing; EF, external fixation

Among the hemorrhage control methods used for the 97 patients with pelvic fractures and hemodynamic instability in this study, the most frequent was angiography, which was performed for 47 patients (48.45\%) in each group. Among these, 32 patients (32.98\%) underwent AE. PPP was the second most frequently used method (45 patients; $46.39 \%)$. Nineteen patients (19.58\%) underwent EF. A total of 17 patients were treated with angiography and PPP; among them, six patients underwent angiography, PPP, and EF. Three patients underwent all three methods. Seven patients underwent PPP and EF.

There were no statistically significant differences in mortality among the hemorrhage control methods used for the 28-day and acute hemorrhage groups, except for EF of the acute hemorrhage mortality group $(p=0.001)$. However, a comparison between the group that underwent any of the three 
190

aforementioned hemorrhage control methods and the group that did not undergo any one of them showed a significant difference in acute hemorrhage mortality between the survival and non-survival groups $(p=$ $0.032)$

Table 4-2 shows the results of the analysis of mortality differences among the hemorrhage control methods. As a result, there were no significant differences between 28-day mortality and acute hemorrhage mortality among the hemorrhage control methods.

\section{Logistic regression analysis of predictors of mortality for hemodynamically unstable patients with}

\section{pelvic fracture}

Results of the univariate and multivariate regression analyses are shown in Table 5-1 and Table 5-2.

Table 5-1 Multivariable regression analysis of risk factors for 28-day mortality

Multivariate

Univariate analysis

analysis

Characteristics

\begin{tabular}{lllll}
\hline Survival & Non- & $p$ & & $p$ \\
& survival & & OR $(95 \% \mathrm{CI})$ & \\
& $(n=60)$ & & & \\
& & & & \\
& & & & \\
$50.72 \pm 20$ & $66.27 \pm 21.6$ & $<0.001$ & $1.056(1.020,1.093)$ & 0.002
\end{tabular}

Transfusion amount (24-

$\begin{array}{lllll}10.9 \pm 7.1 & 19.1 \pm 13.7 & 0.002 & 1.084(1.015,1.156) & 0.016\end{array}$

hour)

$67.61 \pm$

TRISS (\%)

$34.45 \pm 31.15<0.001$

$0.976(0.956,0.997)$

0.022

31.14

$\mathrm{BE}$

$-6.8 \pm 4.6 \quad-10.1 \pm 6.4 \quad 0.012$

Lactate

$\begin{array}{lll}4.3 \pm 3 & 6.6 \pm 3.2 & 0.001\end{array}$




\begin{tabular}{lccc}
\hline GCS & $11.9 \pm 4.7$ & $9.1 \pm 4.9$ & 0.007 \\
SBP & $96.6 \pm 30.4$ & $70.5 \pm 40.9$ & 0.001
\end{tabular}

$\overline{\mathrm{OR} \text {, odds ratio; CI, confidence interval; TRISS, trauma and injury severity score; BE, base excess; GCS, Glasgow }}$ coma score; SBP, systolic blood pressure

Table 5-2 Multivariable regression analysis of risk factors for acute hemorrhage mortality

\begin{tabular}{|c|c|c|c|c|c|}
\hline \multirow[b]{2}{*}{ Characteristics } & \multicolumn{3}{|c|}{ Univariate analysis } & $\begin{array}{l}\text { Multivariate } \\
\text { analysis }\end{array}$ & \multirow[b]{2}{*}{$p$} \\
\hline & $\begin{array}{l}\text { Survival } \\
(n=60)\end{array}$ & $\begin{array}{l}\text { Non- } \\
\text { survival } \\
(n=37)\end{array}$ & $p$ & OR $(95 \% \mathrm{CI})$ & \\
\hline Age & $53.91 \pm 20.8$ & $63.39 \pm 23.4$ & 0.053 & $1.050(1.014,1.087)$ & 0.006 \\
\hline $\begin{array}{l}\text { Transfusion am } \\
\text { hour) }\end{array}$ & $11.5 \pm 7.9$ & $20 \pm 14.3$ & 0.006 & $1.094(1.025,1.169)$ & 0.007 \\
\hline TRISS (\%) & $\begin{array}{l}64.57 \pm \\
31.44\end{array}$ & $31.27 \pm 32.21$ & $<0.001$ & & \\
\hline $\mathrm{BE}$ & $-6.9 \pm 4.6$ & $-10.7 \pm 6.7$ & 0.013 & & \\
\hline Lactate & $4.4 \pm 3$ & $7 \pm 3.2$ & $<0.001$ & & \\
\hline GCS & $11.9 \pm 4.5$ & $8.2 \pm 5.1$ & 0.001 & $0.852(0.740,0.981)$ & 0.026 \\
\hline SBP & $94.4 \pm 29.9$ & $67.6 \pm 45.3$ & 0.007 & $0.978(0.960,0.997)$ & 0.025 \\
\hline
\end{tabular}


Age (OR, 1.056; 95\% CI, 1.020-1.093; $p=0.002)$, lower TRISS (OR, 0.976; 95\% CI, 0.956-0.997; $p=0.022)$, and large amounts of blood transfused within the first 24 hours (OR, 1.084; 95\% CI, 1.015-1.156, $p=0.016$ ) were identified as independent risk factors for death within 28 days. The different hemorrhage control methods did not significantly affect mortality among these patients.

Age $(\mathrm{OR}, 1.050 ; 95 \% \mathrm{CI}, 1.014-1.087 ; p=0.006)$ and a large amount of blood transfused within the first 24 hours (OR, 1.094; 95\% CI, 1.025-1.169; $p=0.007)$ were identified as independent predictors of death with acute hemorrhage. The GCS score (OR, 0.852; CI, 0.740-0.981; $p=0.026)$ and SBP (OR, 0.978; CI, 0.960-0.997; $p=0.025)$ were also independent risk factors for acute hemorrhage mortality of pelvic fracture patients with hemodynamic instability.

\section{Discussion}

\section{Effects of the different hemorrhage control methods on survival}

To understand the nature of hemodynamic instability in pelvic fractures, the most common causes should be known. There are three major sources of bleeding in pelvic fractures: the surface of the fractured bones, the pelvic venous plexus, and arterial injury. Bleeding in pelvic fractures occurs in approximately $90 \%$ of venous cases and approximately $10 \%$ of arterial cases [10]. However, arterial bleeding is more common in cases of ongoing bleeding or hemodynamic instability despite adequate treatment. Although the pelvic capacity during the steady state is only $1.5 \mathrm{~L}$, a pelvic fracture may result in hemodynamic instability because the actual bleeding from the pelvic fracture into the retroperitoneal space can amount to 3-5 L [11]. Therefore, fast and adequate treatment of hemodynamically unstable patients with pelvic fractures is critical.

Based on these pathophysiological considerations, various hemorrhagic control methods are currently used. Among them, the most commonly used methods worldwide are AE, PPP, and EF. According to a recent study performed in the United States, angiography was performed for $42.6 \%$, EF was performed for $36.8 \%$, and PPP was performed for $13.2 \%$ of hemodynamically unstable patients with 
pelvic fractures [6]. Depending on the geographic location or institution, there are differences in procedure applications, priorities, equipment, and available human resources. However, because our study also showed a significant difference between groups with and without hemorrhage control methods, there is no doubt that these three methods have important roles in hemorrhage control in hemodynamically unstable patients with pelvic fractures. There are many ongoing studies about how to optimally apply these methods.

The three individual hemorrhage control methods investigated during this study did not result in significant differences in survival between patient groups. However, a comparison indicated that the groups that underwent any of the hemorrhage control methods had better survival rates than the groups that underwent none.

When interpreting these results, the characteristics of patients with traumatic pelvic fractures, especially those with hemodynamic instability, should be considered. Most fractures sustained by these patients are caused by blunt injuries, and most of them have injuries to other body organs in addition to pelvic fractures. In fact, our survey also showed significant scores for the abdomen and chest. There was also a tendency for increased mortality with combined laparotomy. Therefore, heterogenicity of this injury site was a limitation of this study, and it was difficult to accurately analyze the effects on the survival rates for hemorrhagic control methods. In other words, for patients with pelvic fractures, most are accompanied by various other bodily damage, which may affect early mortality. Therefore, it can be said that the application of appropriate treatments tailored to each patient's situation and the resources of the institution are more important than the concerns about prioritizing specific procedures.

\section{Independent risk factors for mortality in patients with pelvic fracture and hemodynamic instability}

We investigated the risk factors for death for 28-day mortality and mortality related to acute hemorrhage. For 28-day mortality, the most significant cause of death was acute hemorrhage (28/37; 78\%). All other patients except for three who died from multi-organ failure or sepsis died within 7 days. 
In our analysis, we found several independent risk factors for death for hemodynamically unstable patients with pelvic fractures. However, these risk factors were slightly different between the 28-day mortality group and mortality caused by acute hemorrhage group.

Old age was revealed as a common independent risk factor for 28-day mortality (OR, 1.056; 95\% CI, $1.020-1.093 ; p=0.002)$ and acute hemorrhage mortality (OR, 1.050; 95\% CI, 1.014-1.087; $p=0.006)$. Generally speaking, among elderly patients, trauma occurs less often than it does in young people; however, when it occurs, the extent of injury and mortality are considerably higher [12]. The same is true for traumatic pelvic fractures. Studies have shown different cut-off values for age; however, in general, older individuals are at greater risk for severe bleeding from a pelvic fracture than younger individuals. For example, blood vessels show sclerotic changes, meaning that arterial injury may occur even with relatively small trauma forces [12, 13]. Additionally, because compensation mechanisms in older patients are limited compared to those in younger patients, shock can easily occur, even with limited amounts of bleeding. Furthermore, elderly patients are more likely to respond less effectively than younger patients to the same resuscitation methods. This can easily lead to multi-organ failure or subsequent deterioration of immune function and death from sepsis. Various underlying diseases associated with aging also adversely affect the damage caused by pelvic fractures [14]. Therefore, hemodynamically unstable older patients with pelvic fractures require more intensive care.

We also found that the greater the transfusion need within 24 hours, the lower the survival rate of hemodynamically unstable patients. The multivariate analysis revealed transfusion requirements as a common independent risk factor for 28-day mortality (OR, 1.084; 95\% CI, 1.015-1.156; $p=0.016)$ and acute hemorrhage-induced mortality (OR, 1.094; 95\% CI, 1.025-1.169; $p=0.007)$. The need for many blood transfusions means that bleeding is significant. Instability and poor vital signs in pelvic fractures are caused by massive bleeding of more than $2 \mathrm{~L}$. Massive bleeding leads to decreased hemoglobin levels and cardiac output, thereby causing poor oxygen supply to peripheral tissues and eventually resulting in organ 
commonly referred to as the lethal triad [16]. Resuscitation attempts to end this complex series of cascades include a massive transfusion to rescue the patient. Massive transfusions have many side effects, such as transfusion-related acute lung injury, increased infection risk due to immune suppression, hypothermia, coagulopathy, and acidosis. Therefore, massive transfusion protocols have been developed to reduce the transfusion volume to achieve sufficient resuscitation while minimizing side effects. Several guidelines recommend that every institution should have a massive transfusion protocol [17-19]. Recently, methods for optimizing transfusions and limiting the side effects through analysis methods such as thromboelastography have been studied and may help reduce the mortality caused by transfusion requirements in the future [20].

The TRISS was identified as an independent risk factor for 28-day mortality (OR, 0.976; 95\% CI, 0.9560.997; $p=0.022$ ). The TRISS indicates the probability of survival and reflects both the ISS and RTS. In other words, it is an ideal trauma scoring system that reflects both the degree of anatomic damage and pathophysiological state of the injured patient [21]. Therefore, recording these scores for patients with pelvic fractures seems appropriate [22]. Our study showed a marked difference in both the acute hemorrhage group and 28-day mortality group, which had a more than 30\% lower TRISS compared with that of the survival group. Furthermore, the TRISS can be easily calculated during the initial evaluation of the injured patient. Patients with a low TRISS should be considered at high risk and in need of rapid hemorrhage control. The rapid application of treatment based on the TRISS will help improve the survival of hemodynamically unstable patients.

In general, it is accepted that trauma patients with $\mathrm{SBP}<90 \mathrm{mmHg}$ are experiencing hypotension. Recent studies have suggested that an initial SBP in the range of $90-110 \mathrm{mmHg}$ or less in a trauma patient may be indicative of hypoperfusion and is associated with poor patient outcomes [4, 23, 24]. Although different SBP cutoff values were utilized, previous studies have reported that decreased SBP is an independent risk factor for mortality of patients with pelvic fracture $[4,25,26]$. In this study, low SBP and low GCS scores were also identified as independent risk factors for acute hemorrhage mortality with 
traumatic pelvic fractures. Just as massive transfusion is an independent risk factor for these patients, low blood pressure and altered mental status can also be associated with massive heavy bleeding, inadequate response to resuscitation, or hypoperfusion of peripheral organ tissues, including the brain. Therefore, blood pressure and mental status as early predictors of pelvic fracture due to trauma can be important factors for determining the future prognosis of the patient.

\section{Study limitations}

This study had some limitations. First, it was a retrospective study. Second, it used data from two different institutions and included different treatment protocols, different equipment, different medical staff policies, and, consequently, different trauma treatments. Furthermore, differences in patient characteristics and trauma mechanisms due to regional differences should be considered. It should also be remembered that data regarding treatment during the pre-hospitalization phase were missing. Information regarding the records of 119 treatments performed in the ambulance and the exact trauma mechanism were missing. Furthermore, the time of the accident or the time from the accident to hospital admission are often missing, and the amounts of fluid or blood administered before admission are not clearly recorded. Therefore, further studies are needed. This study also did not analyze newer techniques used for hemodynamically unstable patients with pelvic fractures, such as resuscitative endovascular balloon occlusion. In future studies, data regarding more cases and clearly defined and standardized treatment protocols, including the more recent techniques, should be used to yield more specific and useful findings.

\section{Conclusions}

We found that rapid and appropriate application of hemorrhage control methods such as angiography, PPP, and EF can reduce acute hemorrhage-related mortality in hemodynamically unstable patients with pelvic fractures. However, none of the hemorrhage control methods showed any superiority in improving the mortality of pelvic fracture patients who are hemodynamically unstable. Therefore, the application of the appropriate hemorrhage control method for each patient's clinical situation or medical institution's 
330

331

332

333

334

335

336

337

338

339

340

341

342

343

344

345

346

347

situation should be prioritized over trying to determine which hemorrhage control method should be applied first. When evaluating the clinical situation of patients, old age, massive transfusion requirements, low TRISS, hypotension, and altered mental status were all independent risk factors for patient mortality. Therefore, more active and intensive care is needed for these patients. In the future, more research should address the appropriate use of various hemorrhagic control methods for patients with traumatic pelvic fractures and hemodynamic instability.

\section{Abbreviations}

\section{AE: Angio-embolization}

AIS: Abbreviated injury scale

APACHE: Acute Physiology and Chronic Health Evaluation

BE: Base excess

CI: Confidence interval

EF: External fixation

GCS: Glasgow coma scale

ISS: Injury severity score

OR: Odds ratio

PPP: Pre-peritoneal pelvic packing

RTS: Revised trauma score

SBP: Systolic blood pressure

TRISS: Trauma and injury severity score

\section{Declarations}


353 This study was conducted in accordance with the Declaration of Helsinki and was approved by the Institutional

354 Review Boards of Severance Hospital, Yonsei University Health System (4-2019-0761) and Wonju Severance 355 Christian Hospital (CR319120), which waived the requirement for the acquisition of informed consent from 356 patients because of the retrospective nature of the study.

\section{Consent for publication}

358 Not applicable.

\section{Availability of data and materials}

360 There is no additional data available to share with the readers. The datasets used and/or analyzed during the current study are available from the corresponding author on reasonable request.

The authors declare that they have no competing interests.

\section{$364 \quad$ Funding}

365 The authors received no specific funding for this work.

\section{Authors' contributions}

367 Conceptualization, JG.L.; methodology, JY.J. and JG.L.; formal analysis, MJ.K. and SH.L.; investigation and data curation, MJ.K.; writing-original draft preparation, MJ.K. and SH.L.; writing-review and editing, MJ.K. and JY.J.; supervision, JG.L.; project administration, JG.L. All authors have read and approved the final version of the manuscript.

\section{Acknowledgements}

The authors are grateful to their colleagues from trauma teams at both institutions for the inspiration and ideas for this study, as well as to all coordinators for their valuable help and cooperation.

\section{References}


376 2. Cryer HM, Miller FB, Evers BM, Rouben LR, Seligson DL. Pelvic fracture classification: correlation with

377 hemorrhage. J Trauma. 1988;28:973-80.

378 3. Flint L, Babikian G, Anders M, Rodriguez J, Steinberg S. Definitive control of mortality from severe pelvic 379 fracture. Ann Surg. 1990;211:703-6; discussion 706-7.

380 4. Toth L, King KL, McGrath B, Balogh ZJ. Factors associated with pelvic fracture-related arterial bleeding 381 during trauma resuscitation: a prospective clinical study. J Orthop Trauma. 2014;28:489-95.

382 5. Costantini TW, Coimbra R, Holcomb JB, Podbielski JM, Catalano RD, Blackburn A, et al. Pelvic fracture 383 pattern predicts the need for hemorrhage control intervention - results of an AAST multi-institutional study. J Trauma 384 Acute Care Surg. 2017;82:1030-8.

385 6. Costantini TW, Coimbra R, Holcomb JB, Podbielski JM, Catalano R, Blackburn A, et al. Current management 386 of hemorrhage from severe pelvic fractures: results of an American Association for the Surgery of Trauma multi387 institutional trial. J Trauma Acute Care Surg. 2016;80:717-23; discussion 723-5.

388 7. American College of Surgeons Committee on Trauma. Advanced Trauma Life Support® (ATLS®) student 389 course manual. 10th ed. Chicago, IL: American College of Surgeon; 2018.

390 8. Young JW, Burgess AR, Brumback RJ, Poka A. Pelvic fractures: value of plain radiography in early 391 assessment and management. Radiology. 1986;160:445-51.

392 9. Alton TB, Gee AO. Classifications in brief: young and burgess classification of pelvic ring injuries. Clin 393 Orthop Relat Res. 2014;472:2338-42.

394 10. Stein DM, O'Toole R, Scalea TM. Multidisciplinary approach for patients with pelvic fractures and 395 hemodynamic instability. Scand J Surg. 2007;96:272-80.

396 11. Velmahos GC, Toutouzas KG, Vassiliu P, Sarkisyan G, Chan LS, Hanks SH, et al. A prospective study on 397 the safety and efficacy of angiographic embolization for pelvic and visceral injuries. J Trauma. 2002;53:303-8.

398 12. McNamara RM, Rousseau E, Sanders AB. Geriatric emergency medicine: a survey of practicing emergency 399 physicians. Ann Emerg Med. 1992;21:796-801.

400 13. Kimbrell BJ, Velmahos GC, Chan LS, Demetriades D. Angiographic embolization for pelvic fractures in 401 older patients. Arch Surg. 2004;139:728-32; discussion 732-3.

402 14. Mandavia D, Newton K. Geriatric trauma. Emerg Med Clin North Am. 1998;16:257-74. 
$403 \quad$ 15. Jarrar D, Chaudry IH, Wang P. Organ dysfunction following hemorrhage and sepsis: mechanisms and 404 therapeutic approaches (review). Int J Mol Med. 1999;4:575-83.

405 16. Sihler KC, Napolitano LM. Complications of massive transfusion. Chest. 2010;137:209-20.

406 17. Malone DL, Hess JR, Fingerhut AJ. Massive transfusion practices around the globe and a suggestion for a 407 common massive transfusion protocol. J Trauma. 2006;60:S91-6.

408 18. Yücel N, Lefering R, Maegele M, Vorweg M, Tjardes T, Ruchholtz S, et al. Trauma Associated Severe Hemorrhage (TASH) Score: probability of mass transfusion as surrogate for life threatening hemorrhage after multiple trauma. J Trauma. 2006;60:1228-37.

411 19. Dente CJ, Shaz BH, Nicholas JM, Harris RS, Wyrzykowski AD, Patel S, et al. Improvements in early mortality and coagulopathy are sustained better in patients with blunt trauma after institution of a massive transfusion protocol in a civilian level I trauma center. J Trauma. 2009;66:1616-24. resuscitation of trauma-induced coagulopathy: a pragmatic randomized clinical trial comparing a viscoelastic assay to conventional coagulation assays. Ann Surg. 2016;263:1051-9. 21. Bergeron E, Rossignol M, Osler T, Clas D, Lavoie A. Improving the TRISS methodology by restructuring age categories and adding comorbidities. J Trauma. 2004;56:760-7. 22. Lau J, Chui KH, Ip KC, Lee KB, Li W. Improved survival with a standardized multidisciplinary 3-in-1 pelvic damage control protocol for hemodynamically unstable pelvic fracture. J Emerg Trauma Care. 2016;1:1-6. 23. Salim A, Teixeira PG, DuBose J, Ottochian M, Inaba K, Margulies DR, et al. Predictors of positive angiography in pelvic fractures: a prospective study. J Am Coll Surg. 2008;207:656-62. fracture: initial therapy guided by markers of arterial hemorrhage. J Trauma. 2003;54:437-43. 2011;202:404-8. pressure of 90-109 mmHg be included? Shock. 2007;27:134-8. 